\title{
Low value cardiac testing and Choosing Wisely
}

\section{R Sacha Bhatia, ${ }^{1,2,3}$ Wendy Levinson, ${ }^{3,4}$ Douglas S Lee ${ }^{2,3}$}

\begin{abstract}
${ }^{1}$ Women's College Hospital Institute for Health Systems Solutions and Virtual Care, Toronto, Ontario, Canada ${ }^{2}$ Peter Munk Cardiac Centre, University Health Network, Toronto, Ontario, Canada ${ }^{3}$ University of Toronto, Toronto, Ontario, Canada

${ }^{4}$ Saint Michael's Hospital, Toronto, Ontario, Canada
\end{abstract}

\section{Correspondence to}

Dr R Sacha Bhatia, Women's College Hospital Institute for Health Systems Solutions and Virtual Care, Institute for Clinical Evaluative Sciences, Division of Cardiology, University Health Network and Women's College Hospital, University of Toronto, 76 Grenville Street, 6th Floor, Toronto, Ontario M5S 1B2, Canada;

sacha.bhatia@wchospital.ca

Received 22 September 2014 Accepted 24 September 2014 Published Online First 10 October 2014

\section{CLinked}

- http://dx.doi.org/10.1136/ bmjqs-2014-003087

\section{CrossMark}

$$
\begin{aligned}
& \hline \text { To cite: Bhatia RS, } \\
& \text { Levinson W, Lee DS. BMJ } \\
& \text { Qual Saf 2015;24:89-91. }
\end{aligned}
$$

Just a few years prior to the famous Institute of Medicine (IOM) reports on safety and quality, an earlier IOM report classified all quality problems in healthcare as falling into three broad categories: underuse, overuse and misuse. ${ }^{1}$ Until recently, however, the focus on quality has been almost exclusively on the underuse and misuse, and overuse has received much less attention. ${ }^{2}$ This focus is particularly surprising, as much of the early work in healthcare quality focused on overuse. In particular, early research done on the geographical variation of healthcare service delivery implied that a significant proportion of healthcare services, such as surgical procedures, were not necessary. ${ }^{34}$ Despite this early research, the majority of quality improvement efforts over the past decade were directed towards improving patient safety and addressing care gaps related to the underuse of health services.

More recently, with healthcare systems worldwide struggling to contain rising costs, overuse of healthcare services is beginning to receive more attention. In an effort at bringing attention to the generally accepted notion that excessive use of low-value care is a contributor to those costs, and that overuse of tests and treatments may lead to potential patient harm, the American Board of Internal Medicine Foundation launched the Choosing Wisely campaign in 2012 . This physiciandesigned and led campaign focuses on developing 'top 5 lists' of tests, treatments and procedures in various specialties that were deemed to be unnecessary and potentially harmful. ${ }^{5}$ This campaign now has 60 US specialty societies, but participation is growing internationally, including Canada, the Netherlands, Italy, Japan and others.

A major question asked by clinicians, health policy experts and payers (either government or health insurers) is: what is the prevalence of low-value care in clinical practice? Colla and colleagues have examined the question in this study. The researchers used administrative data to estimate the prevalence of one of the Choosing Wisely items, pertaining to the ordering of cardiac testing in low-risk patients. The research team used Medicare claims data to define high-risk and low-risk cardiovascular cohorts and assessed the proportion of patients in both the groups who had received a cardiac test (ECG, stress test, echocardiogram, cardiac CT or MRI) without a stated indication. The results highlighted two key findings. First, the prevalence of cardiac testing with no indication was $13 \%$ in the low-risk cohort and 20\% in the high-risk cohort, mostly driven by the ordering of ECG. Second, regional variation was significant, with prevalence rates varying more than threefold between different Hospital Referral Regions. ${ }^{6}$

This well-designed study highlights some of the challenges of measuring the prevalence of low-value testing using administrative data. Administrative data, by its nature, often do not provide the granularity of clinical information that accurately captures the decision making of providers. These challenges are not unique to this study alone, and in fact, the authors made a diligent effort to provide outcome data to justify their categorisation of low-risk and high-risk populations. In a recent paper by Schwartz and colleagues attempting to assess the economic impact of a basket of Choosing Wisely recommendations, the results ranged widely, from $0.6 \%$ to $2.7 \%$ of Gross Domestic Product (GDP) depending on whether a more specific or sensitive coding algorithm was used. ${ }^{7}$ The paper by Schwartz demonstrates the significant variation in defining low-value care using administrative data. While this variation may not appear large at first, the magnitude of costs ranged from US $\$ 1.9$ to US $\$ 8.5$ billion in the Medicare population. Therefore, small differences in the way low-value care is defined and measured may significantly change estimates of the 
impact that low-value care has on the health system. Attempts to measure low-value care should be performed using data that more accurately reflects the clinical circumstances around medical decisions. These data may include clinical sources, such as Electronic Medical Records (EMR), which may be useful for identification of low-value testing for policy makers and health insurers.

A further issue with the study relates to the issue of risk stratification. The authors defined a low-risk cohort as those without known cardiovascular disease or risk factors. However, patients may erroneously be assigned to the low-risk group if risk factors are not systematically assessed-which is not guaranteed in real-world practice. Furthermore, it may have been the case that some providers appropriately used a multivariable risk index, such as the Framingham risk score to determine the need for further cardiac testing. If one risk stratified the patients in this cohort based on the accepted Framingham risk score many of these patients would not qualify as low risk, based on age alone. The fact that the authors found this cohort had a lower event rate than the high-risk cohort is reassuring, but ultimately does not change the fact that given the older age of the study sample, even the low-risk cohort was not truly 'low risk'. Future work examining prevalence rates of non-indicated cardiac testing in the population aged $<65$ years may shed light on a true 'low-risk' population.

Beyond the methodological questions raised by this study, a larger question is raised by the results. Are the results published by Colla et al, worrying or actually reassuring? In the low-risk cohort, the prevalence of non-indicated cardiac testing was measured at $12.8 \%$, but $12.4 \%$ of this care was accounted for by performance of ECGs. There were very low rates of exercise stress testing (0.3\%), echocardiography $(0.25 \%)$ and advanced cardiac imaging $(0.04 \%)$. Since the overall prevalence seems relatively low, and the majority of the low-value care was due to 'low-cost' testing with ECGs, some may argue that it may not be worth the effort to reduce the prevalence of low-value care based on the results reported by Colla and colleagues. However, the authors acknowledge that despite the low direct costs of inappropriate ECG testing, it may lead to downstream testing whose impact on outcomes, additional testing and costs are not known. Furthermore, a more in-depth look at the data, in particular, the degree of regional variation, does suggest there is work to do to reduce low-value care. In some regions of the USA, the rate of non-indicated cardiovascular testing is as high as $28 \%$. Future work on implementation and evaluation of the impact of Choosing Wisely recommendations may need to focus on assessment of local practices and identifying regional outliers to target interventions.

Finally, and importantly, what is the impact that this paper has for the Choosing Wisely campaign, in the
USA and internationally? First, the paper by Colla is one of the first data-driven efforts to quantify the rate of low-value care across a jurisdiction using administrative data and adds significantly to the growing body of literature evaluating the prevalence of overuse. There is significant effort internationally to begin to develop a systematic methodology to evaluate lowvalue care among researchers, and this paper helps inform that methodology. More importantly, however, this paper provides some sobering perspectives to those engaged in the Choosing Wisely movement and researchers and policy makers regarding the challenges of identifying and eliminating overuse. Colla and colleagues' work represents a sophisticated attempt to quantify overuse of common cardiac testing in the country with the world's highest per capita costs of healthcare. The study results suggest that on a large scale, overuse may be difficult to measure accurately and when averaged over several jurisdictions, the variations in overuse may be attenuated. The study did demonstrate that significant regional variations exist and future research will be required to understand the root causes of that variation, be it physician, patient or system-related factors. Once the causes are identified, it may be possible to develop and test interventions to reduce lowvalue care. In the paper by Colla and colleagues, work is the first step on the long road to address the issue of overuse, an area of quality improvement that, to date, is not well understood.

Choosing Wisely, which began as a small, grassroots campaign to bring awareness to patients and doctors about unnecessary testing, has grown into an international movement that has received significant attention from physicians, policy makers and patients. The clear, straightforward message of the Choosing Wisely campaign is likely one of the main reasons it has resonated so broadly. This work does not detract at all from the message of Choosing Wisely, but suggests that the path to reducing low-value care is not straightforward and will be harder than initially thought. We should not be surprised or deterred by this challenge. After the initial call to reduce medical errors by $50 \%$ in 5 years by the Institute of Medicine, progress was slower than hoped for, but progress was still made. ${ }^{8}$ Also, in the past 10 years, we have made significant gains in the area of underuse, while overuse remains an issue. ${ }^{2}$ While we all hope for big gains and quick wins, the usual trajectory of progress is slow, incremental improvements. Just like with underuse and misuse, progress on overuse while slow, will eventually come.

Contributors RSB was the primary researcher and writer of the manuscript. WL provided research and editing assistance to the manuscript. DSL provided research and editing assistance to the manuscript.

Competing interests DSL was supported by a Clinician-Scientist Award from the Canadian Institutes of Health Research (CIHR). 
Provenance and peer review Not commissioned; internally peer reviewed.

\section{REFERENCES}

1 Chassin MR, Galvin RW. The urgent need to improve health care quality. Institute of Medicine National Roundtable on Health Care Quality. JAMA 1998;280:1000-5.

2 Kale MS, Bishop TF, Federman AD, et al. Trends in the overuse of ambulatory health care services in the United States. JAMA Intern Med 2013;173:142-8.

3 Wennberg JE, Fisher ES, Skinner JS. Geography and the debate over Medicare reform. Health Aff 2002;Suppl Web Exclusives: W96-114. doi:10.1377/hlthaff.w2.96
4 Chassin MR, Kosecoff J, Park RE, et al. Does inappropriate use explain geographic variations in the use of health care services? A study of three procedures. JAMA 1987;258:2533-7.

5 Brody H. Medicine's ethical responsibility for health care reform-the Top Five list. N Engl J Med 2010;362:283-5.

6 Colla $\mathrm{CH}$, Sequist TD, Rosenthal MB, et al. Use of non-indicated cardiac testing in low-risk patients: Choosing Wisely. BMJ Qual Saf 2015;24:149-53.

7 Schwartz AL, Landon BE, Elshaug AG, et al. Measuring lowvalue care in medicare. JAMA Inter Med 2014;174:1067-76.

8 Saaddine JB, Cadwell B, Gregg EW, et al. Improvements in diabetes processes of care and intermediate outcomes: United States, 1988-2002. Ann Intern Med 2006;144:465-74. 